\title{
Granule Cell Activation of Complex-Spiking Neurons in Dorsal Cochlear Nucleus
}

\author{
Kevin A. Davis and Eric D. Young \\ Department of Biomedical Engineering and Center for Hearing Sciences, Johns Hopkins University, \\ Baltimore, Maryland 21205
}

\begin{abstract}
Dorsal cochlear nucleus (DCN) principal cells receive, in addition to their well known auditory inputs, various nonauditory inputs via a cerebellar-like granule cell circuit located in the superficial layers of the DCN. Activation of this circuit (granule cell axons make excitatory synapses on the principal cells but also contact inhibitory interneurons that project to the principal cells) produces strong inhibition of the principal cells. Here we investigate the role of cartwheel cells, homologs of cerebellar Purkinje cells, in producing this inhibition. The responses of type IV units (one type of principal cells) and of cartwheel cells were recorded to ortho- and antidromic activation of the granule cells (i.e., by stimulation of their inputs from the somatosensory cuneate and spinal trigeminal nuclei and by direct stimulation of their parallel fiber axons). Cartwheel cells were identified on the basis of recording depth and complex action
\end{abstract}

potential shape. A four-pulse facilitation paradigm (four pulses at $50 \mathrm{msec}$ intervals) was used; this stimulus allows separation of the apparently simple inhibitory somatosensory response of type IV units into a three-component (inhibition-excitationinhibition) response. As expected, cartwheel cells are excited by granule cell activation; the latencies and four-pulse amplitudes of these responses correspond to the properties of the second, long-latency inhibitory component of type IV responses. The source of the first, short-latency inhibitory response is still unknown. Nevertheless, these results show that cartwheel cells convey inhibitory polysensory information to DCN principal cells.

Key words: dorsal cochlear nucleus; granule cells; cartwheel cells; complex spikes; electrical stimulation; somatosensory/ auditory interaction
The dorsal cochlear nucleus (DCN) is organized into a deep region that receives auditory inputs and a superficial region that receives input from a cerebellar-like granule cell circuit (Lorente de Nó, 1981; Mugnaini and Morgan, 1987). A schematic of the circuitry in superficial DCN is shown in Figure 1. Granule cell axons make direct excitatory terminals on the apical dendrites of pyramidal cells, which are one type of DCN principal cell, but they also activate inhibitory interneurons in the superficial layer (Mugnaini et al., 1980; Manis, 1989; Osen et al., 1995; Manis and Molitor, 1996). Most numerous among these interneurons are cartwheel cells (Wouterlood and Mugnaini, 1984), which bear a homology to cerebellar Purkinje cells (Berrebi et al., 1990). Cartwheel cells are glycinergic and perhaps also GABAergic (Osen et al., 1990) and make inhibitory terminals on pyramidal cells and on giant cells, the second DCN principal cell type (Wouterlood and Mugnaini, 1984; Berrebi and Mugnaini, 1991; Golding and Oertel, 1995).

Granule cells in the cochlear nucleus receive inputs from both auditory and nonauditory sources. The main ascending afferent pathway formed by myelinated type I auditory nerve fibers does not terminate in the granule cell domains (Brown and Ledwith, 1990); rather, granule cells receive auditory projections from unmyelinated type II auditory nerve fibers and from various

Received March 19, 1997; revised June 5, 1997; accepted June 10, 1997.

This work was supported by National Institute of Deafness and Other Communication Disorders Grant DC-00979. Dr. Roger Miller recorded the responses of two complex-spiking neurons to somatosensory stimulation; we thank him for allowing us to use his results in this paper.

Correspondence should be addressed to Dr. Kevin Davis, Johns Hopkins University, Department of Biomedical Engineering, 720 Rutland Avenue, 505 Traylor Research Building, Baltimore, MD 21205.

Copyright (C) 1997 Society for Neuroscience $0270-6474 / 97 / 176798-09 \$ 05.00 / 0$ central auditory nuclei (for review, see Weedman et al., 1996). Nonauditory projections to granule cells include afferents from vestibular endorgans (Burian and Gestoettner, 1988) and the somatosensory dorsal column and spinal trigeminal nuclei [medullary somatosensory nuclei (MSN)] (Itoh et al., 1987).

The role of these multimodal inputs in audition is currently unclear; however, single unit studies in vivo indicate that activation of the somatosensory input produces powerful inhibition of DCN principal cells. Natural somatosensory stimulation, particularly involving movement of the pinna, as well as electrical stimulation in the MSN, can inhibit the spontaneous activity of DCN principal cells for tens of milliseconds (Young et al., 1995). In vitro evidence suggests that cartwheel cells are a likely candidate to mediate this inhibition (Zhang and Oertel, 1994; Golding and Oertel, 1995, 1996). So far, however, there is no evidence that connects the inhibition by somatosensory inputs with the cartwheel cells.

Stimulation of the somatosensory inputs to DCN offers an effective way to activate the granule cell-associated circuits and study their effects on DCN principal cells. Using a four-pulse facilitation paradigm with near-threshold electrical stimuli, Young et al. (1995) showed that the apparently simple monophasic inhibitory response described above can be decomposed into three components (see Fig. 4A): (1) a short-latency inhibitory component; (2) a transient excitatory component; and (3) a longlatency inhibitory component that follows the excitatory component. All three components are seen with orthodromic stimulation of the granule cells through the MSN, but only the latter two are seen with antidromic stimulation of granule cell axons [parallel fibers (PFs)] (Davis et al., 1996). This result suggests that the two inhibitory components are generated by different mecha- 


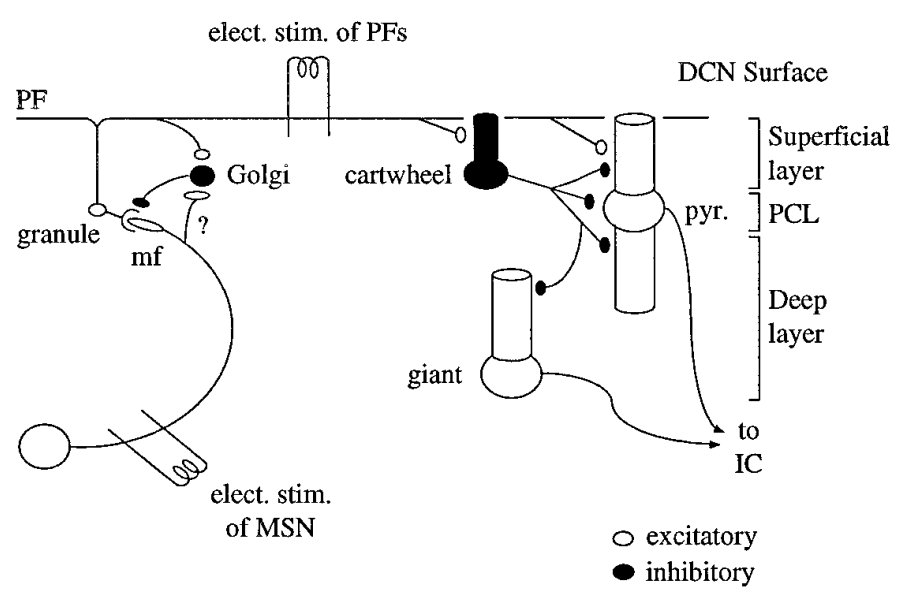

Figure 1. Schematic of the relevant DCN circuitry and somatosensory inputs. The two stimulation sites used in this paper are indicated. The layers of the DCN (identified at right) are defined by the dendrites of the bipolar pyramidal ( $p y r$.) cell. $P C L$ is the pyramidal cell body layer. Pyramidal and giant cells are the DCN principal cell types; their axons project to the inferior colliculus $(I C)$. The deep layer contains the giant cells, the basal dendrites of the pyramidal cells, and a mainly auditory neuropil, which is not shown. The superficial layer receives axons from granule cells $(P F)$ in DCN (shown) and in other parts of the cochlear nucleus (not shown) (Mugnaini et al., 1980). PFs make excitatory terminals on pyramidal cells (and perhaps giant cells; not shown) and on inhibitory interneurons, including Golgi, stellate (not shown), and cartwheel cells. Cartwheel cells are the subject of this paper; their axons terminate on both principal cell types as well as on other cartwheel cells (not shown) (Berrebi and Mugnaini, 1991; Golding and Oertel, 1995). Some of the mossy fiber ( $m f)$ inputs to granule cells are from the representation of the pinna in the somatosensory dorsal column/spinal trigeminal nuclei (MSN) (Young et al., 1995; Wright and Ryugo, 1996). Question marks indicate points of uncertainty in the circuitry.

nisms. It seems likely that the excitatory component reflects direct granule cell excitation of the principal cells, but the sources of the inhibitory components are unclear.

In this paper, the responses of presumed cartwheel cells to both somatosensory and PF stimulation are described. The results show that these neurons are excited by both MSN and PF stimulation; the properties of this excitatory response are appropriate to account for the long-latency but not the short-latency inhibitory component of principal cell responses.

\section{MATERIALS AND METHODS}

Surgical preparation and placement of stimulating electrodes. Experiments were conducted on 14 adult male cats (3-4 kg) with clean external ears, intact clear tympanic membranes, and no sign of ear infection. The

Figure 2. Response properties of complex-spiking units in DCN. $A$, Extracellularly recorded simple and complex action potentials from the spontaneous activity of two complex-spiking units; waveform resolution is $100 \mu$ sec. B1, Acoustic calibration of the system for the experiment in which the data in $B 2$ were obtained. Calibration shows sound pressure level $(S P L)$ at $0 \mathrm{~dB}$ of attenuation at frequencies from 3 to $40 \mathrm{kHz}$. B2, Response map for a typical complex-spiking unit; two repetitions of the map are shown. Map shows rate versus frequency plots at six sound levels for responses to $200 \mathrm{msec}$ tone bursts. Scale bar for rate shown at bottom left; rates are computed as the rate during the tone burst minus the rate during the following silent interstimulus interval. Horizontal lines are average spontaneous rate; solid areas indicate excitation, and stippled areas indicate inhibition present in both repetitions. BF, marked with a vertical line at the top of plot, $19.5 \mathrm{kHz}$; depth, $0.38 \mathrm{~mm}$. C, Examples of discharge rate versus sound level curves in response to BF tones (solid lines) and broadband noise (dashed lines) for the four common response types observed. Sound levels in all plots are shown in $\mathrm{dB}$ of attenuation;

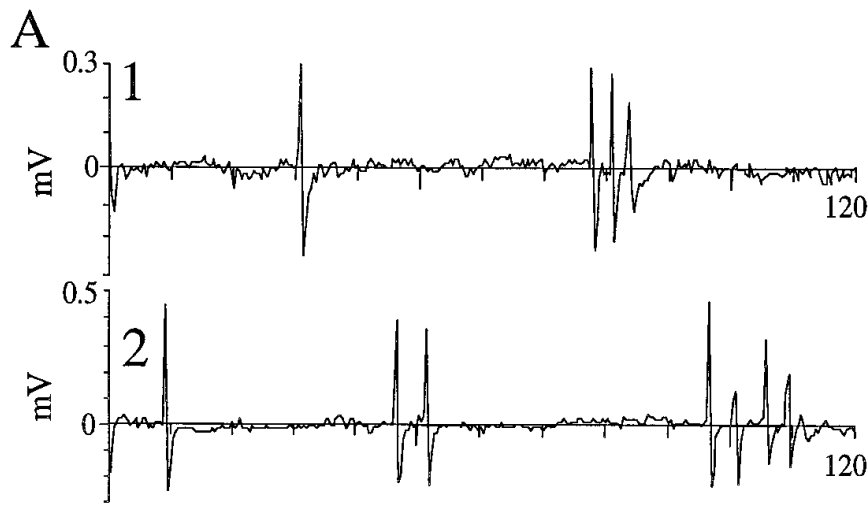

Time, ms

B
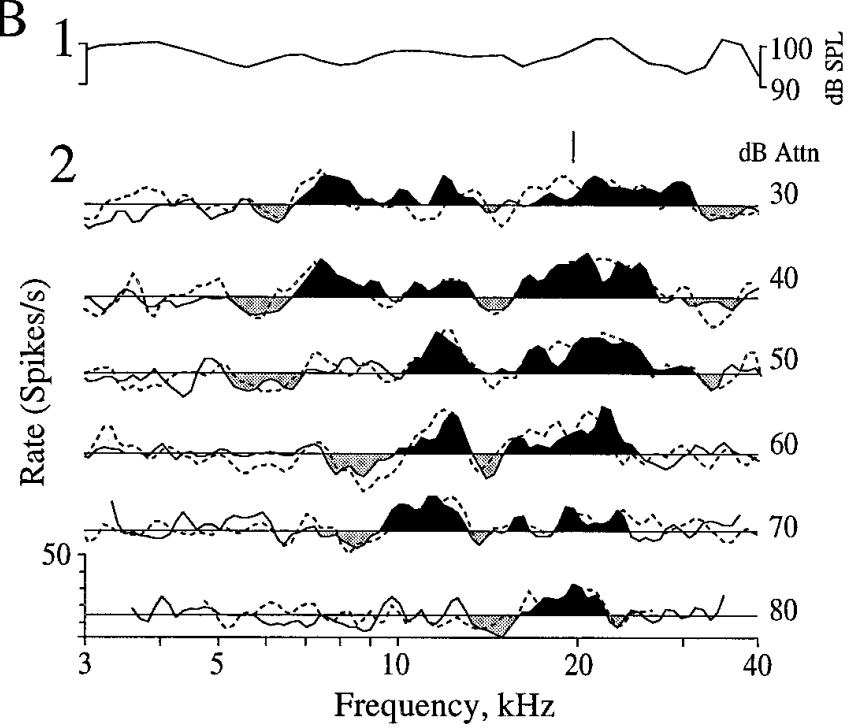

C

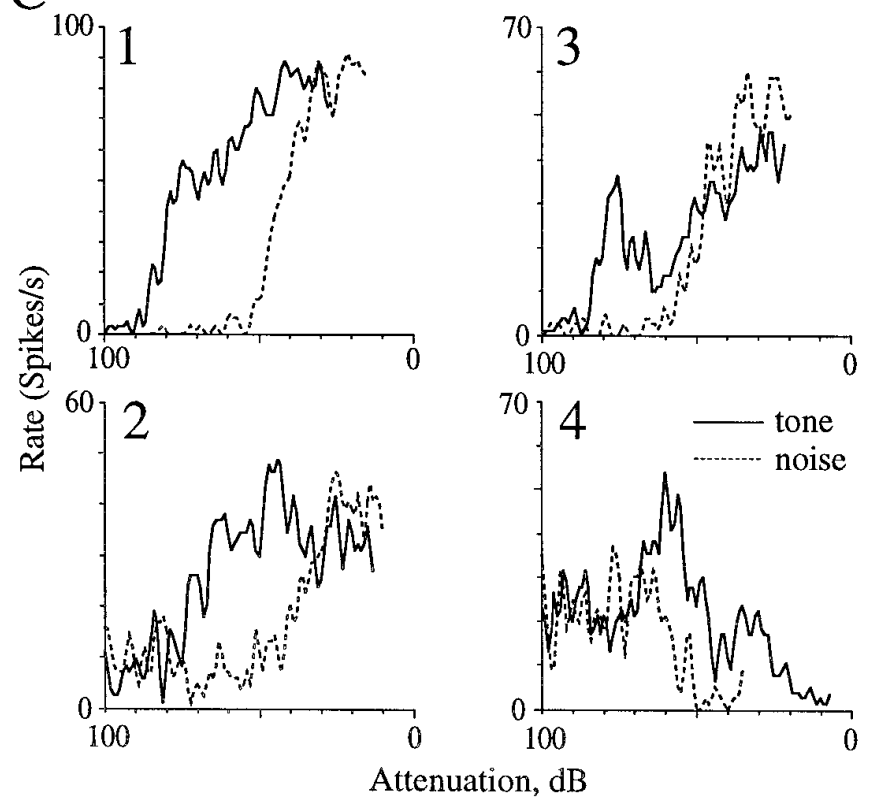

actual SPL varies with the acoustic calibration, but $0 \mathrm{~dB}$ attenuation is near $100 \mathrm{~dB}$ re $20 \mu \mathrm{Pa}$ for $\mathrm{BF}$ tones and near $40 \mathrm{~dB}$ re $20 \mu \mathrm{Pa} / \sqrt{ } \mathrm{Hz}$ for noise spectrum level. Unit BFs and depths are as follows: $C 1,13.6 \mathrm{kHz}, 0.3$ $\mathrm{mm} ; C 2,14.7 \mathrm{kHz}, 0.59 \mathrm{~mm} ; C 3,11.6 \mathrm{kHz}, 0.24 \mathrm{~mm}$; and $C 4,16.8 \mathrm{kHz}$, unknown. 
Table 1. Properties of complex-spiking units

\begin{tabular}{lllll} 
Depth $(\mu \mathrm{m})$ & $\begin{array}{l}\text { Interspike interval } \\
\text { within a burst }(\mathrm{msec})\end{array}$ & $\begin{array}{l}\text { Spontaneous } \\
\text { rate }\end{array}$ & $\begin{array}{l}\text { Maximum rate } \\
\text { to BF tones }\end{array}$ & $\begin{array}{l}\text { Maximum rate } \\
\text { to noise }\end{array}$ \\
\hline $360 \pm 131$ & $3.4 \pm 0.6$ & $13.4 \pm 9.1$ & $74.3 \pm 51.8$ & $79.7 \pm 61.5$
\end{tabular}

$n=39$. Table entries, mean \pm 1 SD. Rates are in spikes/sec.

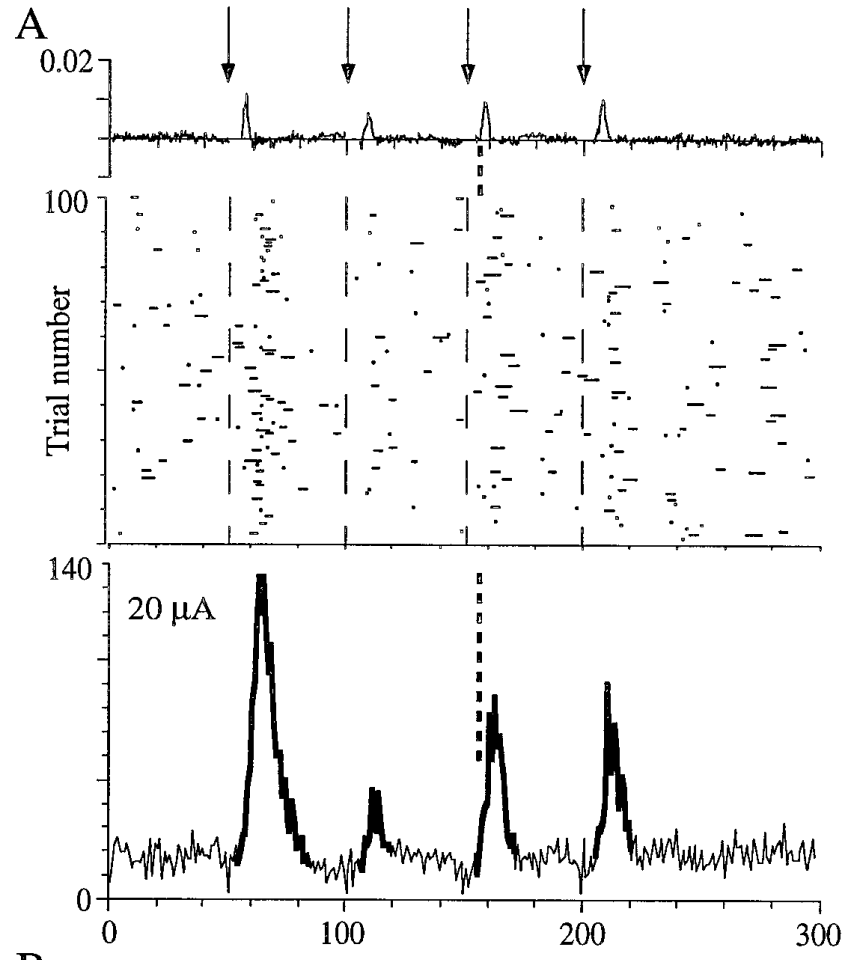

B
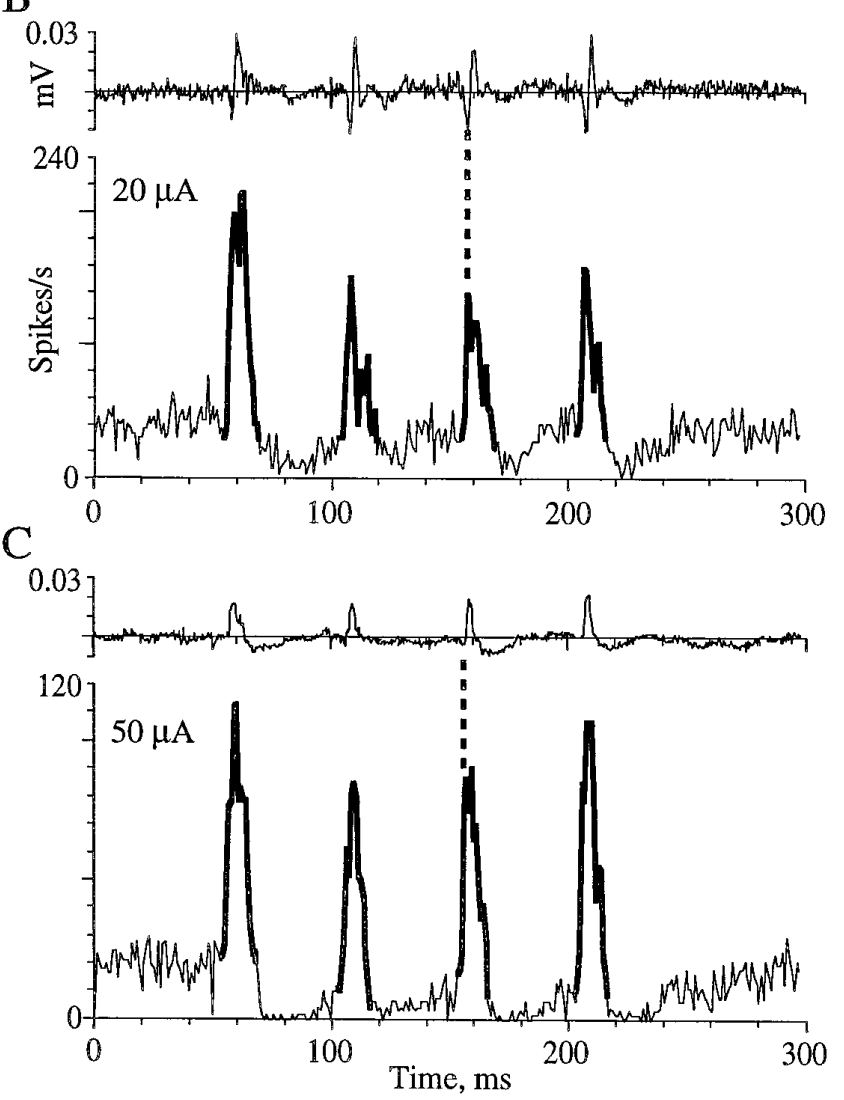

experimental preparation was similar to that described in Davis et al. (1996). Briefly, cats were tranquilized with xylazine (2 mg, i.m.), premedicated with atropine ( $0.1 \mathrm{mg}$, i.m.), and anesthetized with ketamine (initial dose: $40 \mathrm{mg} / \mathrm{kg}$, i.m.; supplemental doses: $15 \mathrm{mg} / \mathrm{kg}$, i.v.). Thereafter, core body temperature was maintained between 38 and $40^{\circ} \mathrm{C}$ using a rectal probe and a feedback-controlled heating blanket. Cats were decerebrated by aspiration through the brainstem rostral to (sparing) the superior colliculus; subsequently, no further anesthetic was given. The cat's head was fixed in the recording position, $35^{\circ}$ nose down with respect to horizontal stereotaxic coordinates, with a headpiece and two earbars. The left pinna was reflected to allow tight coupling of a closed acoustic system (electrostatic driver) (Sokolich, 1977) to the left ear through a hollow earbar (a typical acoustic calibration curve for this system is shown in Fig. 2B1). Despite the damage to the tissue surrounding the pinna, strong responses to touching or moving the left pinna were observed in the MSN of all preparations.

The MSN were exposed at the foramen magnum by removing 3-4 $\mathrm{mm}$ of occipital skull rostral and lateral to the foramen (exposing the underlying obex) and removing the meninges between the skull and atlas. A thin layer of agar was placed over the exposure to prevent drying. The stimulating electrode was advanced into the MSN ( $\sim 3 \mathrm{~mm}$ lateral to the obex) until manual somatosensory stimulation indicated that the receptive field of the background activity was centered on the pinna and adjacent skin. The DCN was exposed by removing the skull and meninges below the nuccal ridge from the midline to the left sigmoid sinus and aspirating the portion of the cerebellum above the DCN. The PF stimulating electrode was placed on the surface of the DCN $\sim 1 \mathrm{~mm}$ medial to and in roughly the same transverse plane as the recording electrode. Before placement of the MSN stimulating electrode and throughout the recording session, cats were paralyzed with gallamine triethiodide (10 $\mathrm{mg} / \mathrm{hr}$, i.v.) to prevent reflex movements and artificially respired at an end-tidal $\mathrm{CO}_{2}$ of $4 \%$. Paralysis was induced no earlier than $4-5 \mathrm{hr}$ after the decerebration to ensure that the decerebration was complete (as judged by lack of voluntary movements).

Stimulating and recording procedures. Electrical stimuli were produced by optically isolated current sources and applied once per second as a train of four bipolar pulses (100 $\mu \mathrm{sec}$ per phase) spaced at $50 \mathrm{msec}$ interpulse intervals. Current amplitudes were 10,20 , or $50 \mu \mathrm{A}$ in the MSN and 100, 200, or $400 \mu \mathrm{A}$ at the DCN surface; the difference in the required current amplitudes could reflect differences in the sizes of axons being stimulated or differences in the coupling between the electrode and the axons. The stimulating electrodes were bipolar tungsten (Microprobe, Garden Grove, CA), with a tip spacing on the order of $50 \mu \mathrm{m}$.

Unit activity was recorded with platinum-iridium metal electrodes. The signal from the recording electrode was amplified and filtered in two channels: (1) evoked potentials (EPs) were digitized at a $10 \mathrm{kHz}$ sampling rate after low-pass filtering the signal at $5 \mathrm{kHz}$, and (2) single units were detected using a variable-threshold Schmitt trigger. Stimulus artifacts were small and brief for MSN stimulation but could be large and

Figure 3. Responses of DCN complex-spiking units to electrical stimulation at a pinna site in the MSN show both excitation (bold) and inhibition. A, Top, EP at recording site; bottom, peristimulus time (PST) histogram of the responses; middle, dot raster of the responses for 100 trials (the complex action potentials have been connected with lines). Complex-spiking neurons typically exhibit some spontaneous activity; the PST histograms show the effects of the stimuli on the spontaneous activity of the units in the absence of acoustic stimuli. Arrows at the top show the times of the four electrical pulses, which were spaced $50 \mathrm{msec}$ apart and presented once per second; the current level is given above the histograms. Heavy vertical dashed lines in the PST histograms are placed at the onset of the EP in response to the third pulse. PST histograms were constructed from 400 repetitions of the stimulus using a binwidth of 1 msec. Unit BFs and depths as follows: $A, 3.5 \mathrm{kHz}, 0.31 \mathrm{~mm} ; B, 7.2 \mathrm{kHz}$, $0.41 \mathrm{~mm}$; and $C, 4.45 \mathrm{kHz}, 0.43 \mathrm{~mm}$. 

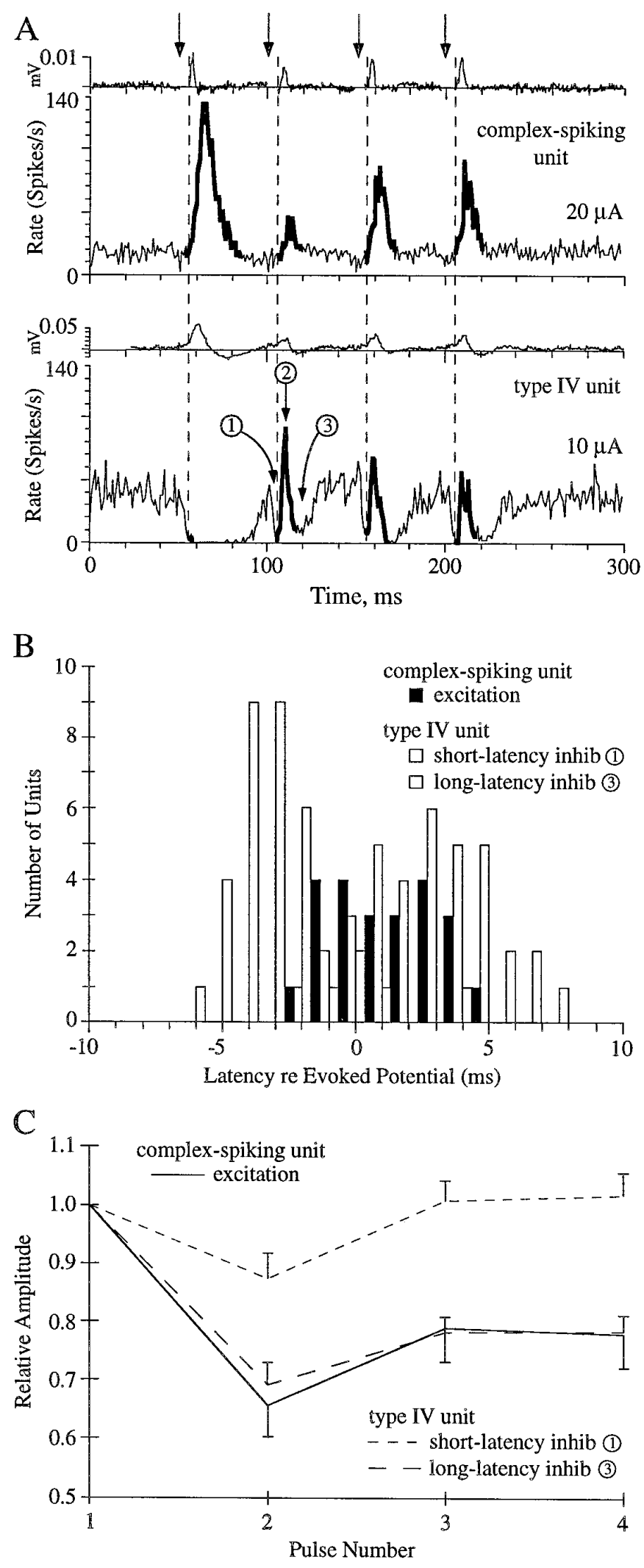

Figure 4. Comparison of complex-spiking and type IV unit response properties to MSN stimulation. $A$, Response of a complex-spiking unit (Fig. $3 A$ ) above the response of a type IV unit from a different experiment (Davis et al., 1996); the circled numbers in the type IV unit response denote the three components of its response: (1) short-latency inhibition; (2) excitation (bold); and (3) long-latency inhibition. The vertical dashed long duration (1-5 msec) for PF stimulation; data recorded where the artifact interfered with triggering at latencies important for interpretation of results were not analyzed further.

Experimental protocol and unit identification. The recording electrode was advanced into the DCN in 1-2 $\mu \mathrm{m}$ steps while $100 \mathrm{msec}$ search tones were presented at the best frequency (BF) of the background activity. Units were characterized by their BFs and by their responses to $200 \mathrm{msec}$ $\mathrm{BF}$ tone and broadband noise bursts presented once per second at sound levels over a $100 \mathrm{~dB}$ range in $1 \mathrm{~dB}$ steps. A unit was presumed to be a cartwheel cell on the basis of the following criteria: (1) it was located in superficial DCN (within the first $0.5 \mathrm{~mm}$ of a track; routine histology to reconstruct tracks was not performed), and (2) it exhibited complex-spike discharges where complex spikes consist of a burst of two to five action potentials, typically with progressively decreasing amplitude. Once a unit was classified, responses to the four-shock electrical stimulation paradigm of the MSN were acquired (typically 100-400 trials), followed by digitization and averaging of the potential evoked in response to the same stimuli at the recording site. Then, responses to the electrical stimulation of the PFs were acquired, followed by recording of the EPs. The isolated unit's action potentials did not significantly affect the shape of the averaged EPs because the responses to both MSN and PF stimulation, although predominantly excitatory, were not well timed. To allow for the detection of inhibitory responses, some background activity is necessary; for units with no spontaneous activity, a background discharge rate was evoked using low-level $\mathrm{BF}$ tones.

\section{RESULTS}

Extracellular single unit responses were obtained from 39 units that exhibited properties consistent with those of cartwheel cells. Most of the units (33/37) were recorded within $500 \mu \mathrm{m}$ of the DCN surface (Table 1) where cartwheel cells are located (Wouterlood and Mugnaini, 1984). Two units that otherwise exhibited cartwheel cell-like characteristics were obtained in medial-to-lateral electrode penetrations for which depth measurements are not available. All of the 39 units exhibited complexspike discharges in addition to simple spikes. Cartwheel cells are the only neurons in cochlear nucleus that have been shown to give complex spikes (Zhang and Oertel, 1993; Manis et al., 1994). Figures $2 A 1$ and $A 2$ show the digitized waveforms of the spontaneous activity from two such complex-spiking neurons. The complex spikes typically consisted of a burst of two to four action potentials whose amplitudes decreased during the burst, although the intraburst spike attenuation was not always orderly (e.g., the second burst in Fig. 2A2). The average interspike interval for spikes within the bursts was $3.4 \mathrm{msec}$ (Table 1), consistent with the value reported for identified cartwheel cells in guinea pig DCN (Manis et al., 1994).

Most of the complex-spiking neurons had broadly tuned re-

lines are aligned with the onsets of the EPs at the recording site of the complex-spiking unit; the onsets of the EPs for the type IV unit are very similar. These two units were chosen to be typical of the data sets; they have rather different BFs $(3.5 \mathrm{kHz}$ for the complex-spiking unit and 0.27 $\mathrm{kHz}$ for the type IV unit), but no features of the MSN responses vary consistently with BF. $B$, Latencies to the excitatory component of complex-spiking units (filled bars) and to the short- (gray bars) and long-latency (open bars) inhibitory components of type IV units. Latencies are relative to the onset of the EP; the onset of a feature is defined to be the time at which the response is midway between the spontaneous rate and the maximum or minimum rate. $C$, Relative amplitudes of the response components (see legend) as a function of MSN stimulus pulse number. This figure shows the average ratio of the amplitudes of these features at pulses 2 through 4 relative to their amplitudes in response to pulse 1. Error bars represent SEM; the numbers of units included are as follows: 24 complex-spiking units; 30 type IV units showing short-latency inhibition; and 31 type IV units showing long-latency inhibition. All averages at pulses $2-4$ are significantly different from $1(p<0.01)$, except for the relative amplitude of the type IV unit short-latency component at pulses 3 and 4 . All curves show a change in slope at the second pulse; the relative amplitude at pulse 4 is significantly different $(p<0.05)$ from that at pulse 2 for all curves. 


\begin{tabular}{|c|c|c|c|c|c|c|}
\hline \multirow[b]{2}{*}{ Stimulus site } & \multicolumn{2}{|c|}{ Complex-spiking units } & \multicolumn{4}{|c|}{ Type IV units } \\
\hline & $\begin{array}{l}\text { Stimulus } \\
\text { to EP }\end{array}$ & $\begin{array}{l}\text { EP to } \\
\text { excitation }\end{array}$ & $\begin{array}{l}\text { Stimulus } \\
\text { to EP }\end{array}$ & $\begin{array}{l}\text { EP to S-L } \\
\text { inhibition }\end{array}$ & $\begin{array}{l}\text { EP to } \\
\text { excitation }\end{array}$ & $\begin{array}{l}\text { EP to L-L } \\
\text { inhibition* }\end{array}$ \\
\hline MSN & $6.8 \pm 1.3$ & $1.0 \pm 2.0^{a, b, c}$ & $6.2 \pm 0.6$ & $-2.5 \pm 2.0^{a}$ & $1.4 \pm 2.4^{b}$ & $2.4 \pm 2.5^{c}$ \\
\hline PFs & $4.9 \pm 1.2$ & $0.3 \pm 1.4^{d, e}$ & $5.4 \pm 1.1$ & & $-0.2 \pm 1.1^{d}$ & $2.7 \pm 1.5^{e}$ \\
\hline \multicolumn{7}{|c|}{$\begin{array}{l}\text { MSN, Medullary somatosensory nuclei; PF, parallel fiber; EP, evoked potential; S-L, short latency; L-L, long latency. } \\
\text { Latencies measured in msec; table entries, mean } \pm 1 \text { SD. }\end{array}$} \\
\hline \multicolumn{7}{|c|}{$\begin{array}{l}\text { * Latency values for type IV units not showing excitation. Values with the same superscripts are compared: }{ }^{a, c, e} \text {, values that } \\
\text { are significantly different }(p<0.01 \text {; two-sided } t \text { test }) ; b, d \text {, values that are not significantly different }(p>0.5) \text {. The stimulus } \\
\text { to EP latencies for MSN and PF stimulation in complex-spiking units are similar to the corresponding values in type IV units } \\
(p>0.3) \text {; within both unit types; however, PF stimulation produces significantly shorter latencies than MSN stimulation } \\
(p<0.05) \text {. }\end{array}$} \\
\hline
\end{tabular}

sponse areas (Fig. 2B2), low spontaneous rates, and weak responses to BF tones and broadband noise (Fig. 2C1-4, Table 1). Classification of these response patterns according to the scheme usually used in cochlear nucleus is difficult because response maps for these units typically show highly variable spontaneous rates, multiple regions of excitation and inhibition, and wide tuning at threshold. The example in Figure $2 B 2$ is typical; two repetitions of the response map are shown by the dotted and solid lines. There is a general correspondence of excitatory (black fill) and inhibitory ( gray fill) areas between the two repetitions, but also considerable variability. Despite some ambiguity in the BF of some units, four distinct types of $\mathrm{BF}$ and noise rate-level response properties were observed, and representative examples are shown in Figure 2C1-4. In the response map scheme (for review, see Young, 1984), these would be classified as type I/III (Fig. 2C1, seen in eight units), type III (Fig. 2C2, 14 units), type IV-T (Fig. $2 C 3$, six units), and type IV (Fig. 2C4, seven units), respectively, with the caveat that all the type IV-like units (e.g., Fig. 2C4) are inhibited by high-level noise. Four units could not be classified because of lack of data. Response type was not correlated with depth of the unit $(p>0.5)$, nor was there a correlation between maximum response rate and depth $(p>0.5)$.

\section{Effects of MSN stimulation on complex-spiking units}

The effects of MSN stimulation were studied for 26 complexspiking units; two units failed to respond to MSN stimulation. Responses from three typical units are shown in Figure 3. For each plot, the top panel shows the potential at the recording site in the DCN evoked by a sequence of four 20 or $50 \mu \mathrm{A}$ shocks delivered to the MSN, and the bottom panel shows a histogram of the responses (100-400 trials) of a single unit that was otherwise firing spontaneously. The middle panel in Figure $3 A$ shows a dot raster of responses for 100 of the trials included in the histogram shown in the bottom panel; the spikes within a complex action potential are connected with lines. The responses show two components: (1) a transient excitatory component (bold) at the onset of the EP (e.g., centered on or near the heavy vertical dashed lines shown at the onset of third EP) that is generated, in large part, from complex spikes; and (2) an inhibitory component that follows the excitatory component, which may (Fig. 3C) or may not (Fig. $3 A$ ) be prominent. Note that the amplitudes of the response components change in a consistent way as a function of the stimulus pulse number; that is, the response components are largest at the first pulse, smallest at the second pulse, and then tend to increase.

A comparison of the response properties of complex-spiking cells and DCN principal cells to MSN stimulation is shown in
Figure 4. All the principal cell data shown in this paper are from type IV units; the type IV response is the most common response type associated with principal cells in decerebrate cat (Young, 1980). In Figure $4 A$, the response of a complex-spiking unit (Fig. $3 A$ ) is positioned above the response of a type IV unit from a different experiment (Davis et al., 1996). The vertical dashed lines are aligned with the onsets of the EPs recorded at the site of the complex-spiking unit; the latencies to the onsets of the EPs at the type IV unit recording site are similar (columns 2 and 4 in Table 2). The histogram of the type IV unit shows the three response components mentioned previously: (1) short-latency inhibition, (2) excitation (bold), and (3) long-latency inhibition. Note that for each stimulus pulse, the onset of the excitatory response of the complex-spiking unit coincides in latency with the onset of the excitatory component of the type IV unit response; that is, the excitatory component of the complex-spiking unit follows the short-latency inhibitory component in the type IV unit response, but precedes its long-latency inhibitory component. Also note that the pattern of response amplitudes of the excitatory response of the complex-spiking unit mirrors the pattern of the long-latency inhibitory component of the type IV unit response. In both cases, the largest response is seen for the first stimulus pulse, the smallest is seen for the second, and there is a small increase in amplitude for the third and fourth pulses.

These comparisons are quantified in Figure 4, $B$ and $C$. Figure $4 B$ shows a histogram of the latencies to the onset of the excitatory response of complex-spiking units, and of the latencies to the onset of the short- and long-latency inhibitory response components of type IV units to MSN stimulation (data from Davis et al., 1996). Here, the onset of a response component is defined to be the time where the average spike rate is at the midpoint between the spontaneous rate and the maximum or minimum rate. The latency to excitation or inhibition is the time of this midpoint relative to the onset of the EP averaged across the responses to the four pulses of the stimulus. For complex-spiking units (solid bars), the average latency of excitation is $1.0 \pm 2.0 \mathrm{msec}$ (mean \pm $1 \mathrm{SD}$ ), which is near the onset of the EP. This value is not significantly different from the mean latency of the excitatory component in type IV units $(1.4 \pm 2.4 \mathrm{msec})$; however, the mean latency of the excitatory response of the complex-spiking units is significantly greater than the mean latency to the short-latency inhibitory response of type IV units (gray bars; $-2.5 \pm 2.0 \mathrm{msec}$ ), but significantly less than the mean latency to the long-latency inhibitory response of type IV units (open bars; $2.4 \pm 2.5 \mathrm{msec}$ ). The latency data are summarized in Table 2.

Figure $4 C$ compares the four-pulse amplitude changes of the 
excitatory response of complex-spiking units to that of the shortand long-latency inhibitory response components of type IV units. Average amplitudes of the component are shown as a function of the pulse number normalized by their values at the first pulse (type IV units data from Davis et al., 1996). Amplitudes were measured as the peak amplitude of the feature minus the spontaneous activity of the unit. In cases in which the inhibitory response saturated at zero for type IV units, as in Figure $4 A$, the saturated value was used; however, if the second or later responses also saturated, then the data were not used (five cases). The short- and long-latency components were identified by latency in cases with very small excitatory peaks, as in Figure $4 A$. All three response components show similar behavior: they are maximal at the first pulse, decrease at the second pulse, and then increase slightly. At each pulse beyond the first, the relative amplitude of the excitatory response of complex-spiking units (solid line) is comparable to that of the long-latency inhibitory component of type IV units (long-dashed line; $p>0.5$ ), but is significantly smaller than that of the short-latency component of type IV units (short-dashed line; $p<0.01$ ).

\section{Effects of PF stimulation on complex-spiking units}

The effects of a train of electric shocks of the DCN PFs were studied for 17 complex-spiking units; responses were observed in 15 of these (two others failed to respond). The responses of complex-spiking units to PF stimulation were generally similar to those produced by MSN stimulation. The major differences are that shocks to the PFs typically elicited only simple spikes and that the pattern of change of response amplitudes with pulse number were different. Responses from three typical units are shown in Figure 5. The layout of this figure is the same as Figure 3; stimulus artifacts have been removed from all plots in the regions where the EP plots are broken. The responses show two components: (1) a transient excitatory component (bold) at the onset of the EP (e.g., centered on or near the heavy vertical dashed lines shown at the onset of third EP); and (2) an inhibitory component that follows the excitatory component, which may (Fig. $5 C$ ) or may not (Fig. 5A) be prominent. In contrast to the responses to MSN stimulation (Fig. 3), direct PF stimulation typically elicits only simple spikes from complex-spiking units, even at the fourth pulse, where the units respond most strongly. For comparison, the fraction of complex spikes evoked by the fourth stimulus pulse averaged $31 \%$ for MSN stimulation but $2 \%$ for PF stimulation. A second noticeable difference between PF and MSN responses is that the amplitudes of both the excitatory and inhibitory components in the PF response usually increase monotonically as a function of pulse number.

A comparison of the response properties of complex-spiking units and DCN principal cells (type IV units) to PF stimulation is shown in Figure 6. In Figure $6 A$, the response of a complexspiking unit (Fig. $5 A$ ) is positioned above the response of a type IV unit from a different experiment (Davis et al., 1996). The type IV unit shows two response components: (1) excitation (bold) followed by (2) inhibition. Similar to the MSN case, the excitatory response of the complex-spiking unit, the presumed inhibitory interneuron, coincides with the type IV unit excitatory response and precedes the long-latency inhibitory response of the type IV unit. Also, the pattern of amplitude change with pulse number of the excitatory response of the complex-spiking unit mirrors the pattern of the inhibitory response of the type IV unit. In both cases, the responses monotonically increase in amplitude throughout the four pulses of the stimulus.

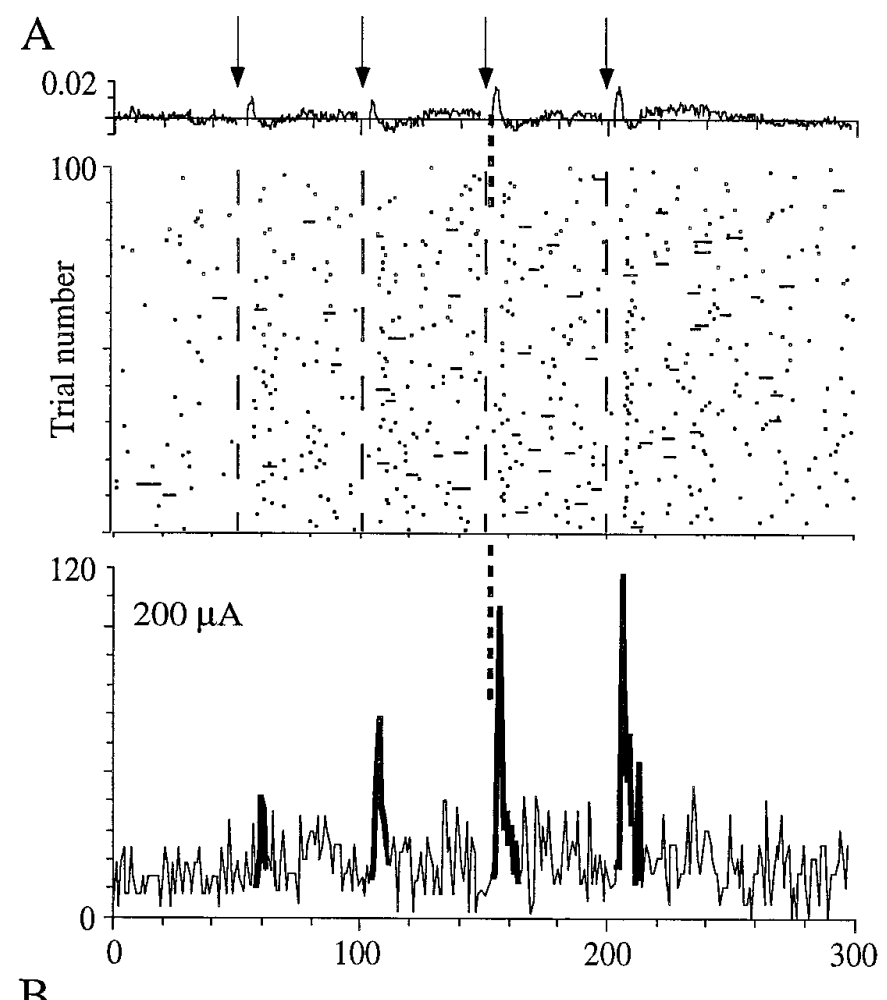

$\mathrm{B}$

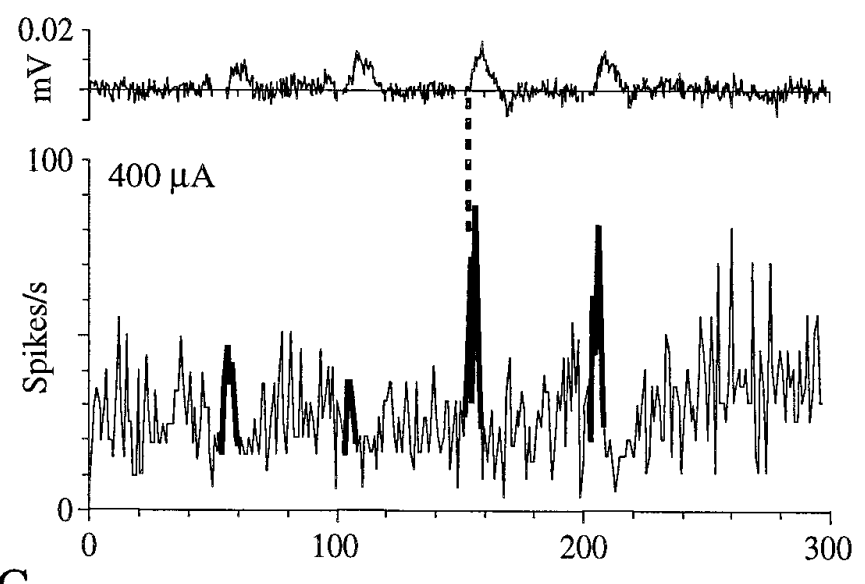

$\mathrm{C}$

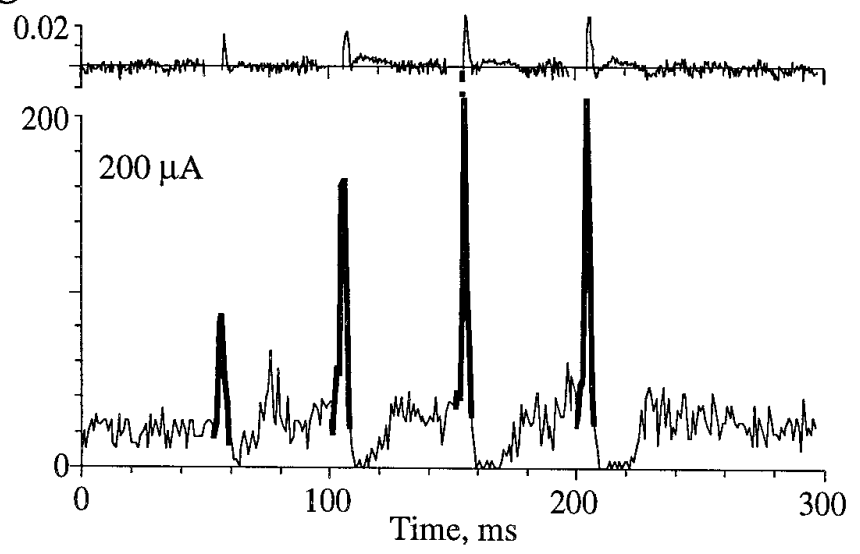

Figure 5. Responses of DCN complex-spiking units to electrical stimulation of DCN PFs also show both excitation (bold) and inhibition. The organization of this figure is the same as for Figure 3. Unit BFs and depths as follows: $A, 18.0 \mathrm{kHz}, 0.28 \mathrm{~mm} ; B, 10.5 \mathrm{kHz}, 0.18 \mathrm{~mm}$; and $C, 12.3$ $\mathrm{kHz}, 0.29 \mathrm{~mm}$. 

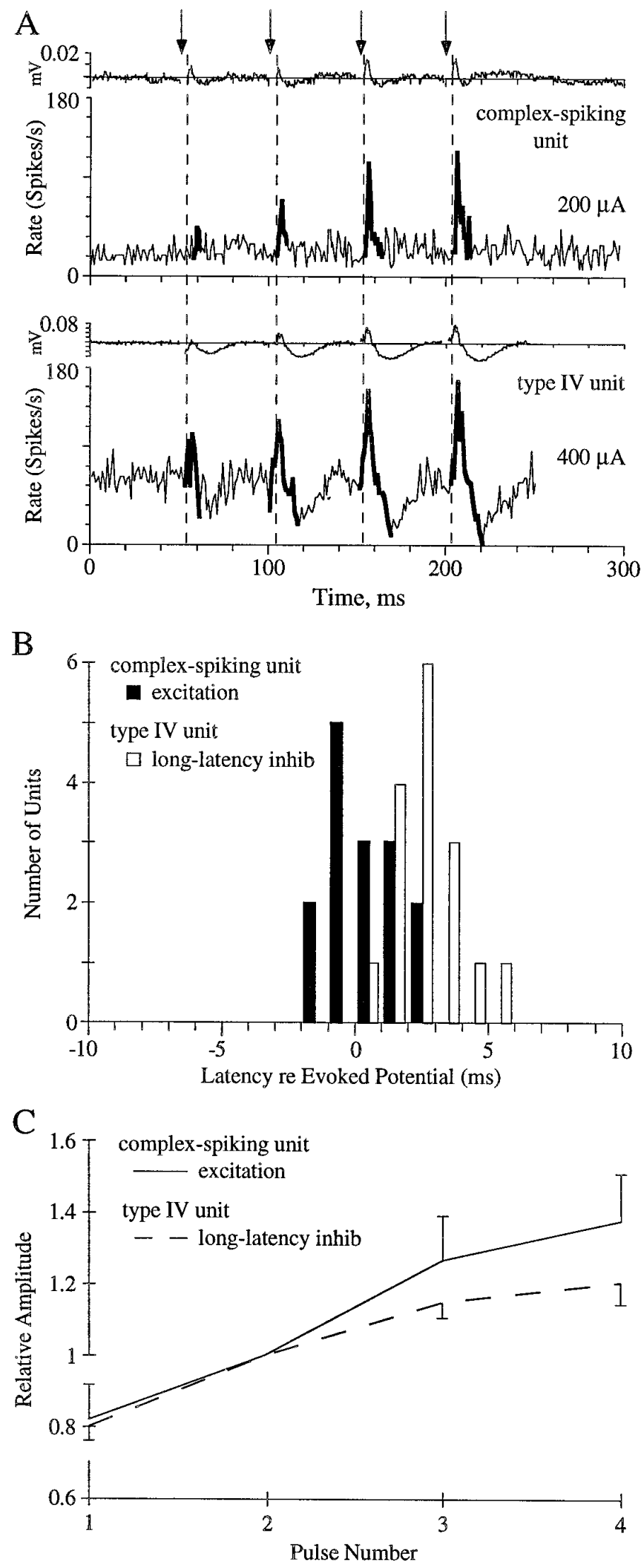

Figure 6. Comparison of complex-spiking and type IV unit response properties to PF stimulation. The organization of this figure is the same as for Figure 4. $A$, Response of a complex-spiking unit (Fig. 5A) above the response of a type IV unit from a different experiment (Davis et al., 1996); the type IV unit response shows two components: excitation (bold) followed by inhibition. These two units are typical of the data sets (unit BFs, $18.0 \mathrm{kHz}$ for the complex-spiking unit and $4.3 \mathrm{kHz}$ for the type IV
Figure $6 B$ shows a histogram of the latencies to the onset of the excitatory response of complex-spiking units, and of the latencies to the onset of the long-latency inhibitory response of type IV units to PF stimulation (data from Davis et al., 1996). For complex-spiking units (solid bars), the average latency of excitation is $0.3 \pm 1.4 \mathrm{msec}$, corresponding to the onset of the EP. This value is comparable to the mean latency of excitation in type IV units $(-0.2 \pm 1.1 \mathrm{msec}$ ) (Table 2). As was the case for MSN stimulation, the mean latency of the excitatory response of the complex-spiking units is significantly less than the mean latency to the long-latency inhibitory response of type IV units (open bars; $2.7 \pm 1.5 \mathrm{msec}$ ). The difference, however, appears more distinct here because there is less scatter in these data. Presumably, PF stimulation produces a well defined volley of action potentials with similar latencies in all PFs, whereas MSN stimulation produces a more dispersed volley because of the dispersed nature of the somatosensory projection to the DCN (Itoh et al., 1987).

Figure $6 C$ compares the pattern of the four-pulse responseamplitude changes of the excitatory response of complex-spiking units with that of the inhibitory response of type IV units. Here, the average amplitudes of the component are normalized by their values at pulse 2 because of the small size of the responses to pulse 1, which leads to unreliable results. As a function of pulse number, the amplitudes of the components increase together; no reversal of the trend is observed at the second pulse as is observed in response to MSN stimulation. The relative amplitudes are statistically indistinguishable $(p>0.3)$ at each pulse in the stimulus.

\section{DISCUSSION}

Complex-spiking neurons are excited by both MSN and PF stimulation. The latency and pattern of four-pulse amplitude change of this excitation are appropriate to account for the long-lasting long-latency inhibition exhibited by type IV units in response to stimulation at these two sites. In response to MSN stimulation only, type IV units also show short-latency inhibition; however, the excitatory response of complex-spiking units has a latency longer than this component and therefore cannot mediate it. Complex-spiking units also exhibit an inhibitory component after their excitatory response (e.g., Figs. 3C, 5C); this component resembles the long-latency inhibition of type IV units in most of its properties (not shown). This response could result from active inhibitory processes, such as mutual inhibition among cartwheel cells (Osen et al., 1990; but see Golding and Oertel, 1996, who show that the interaction among cartwheel cells is not always inhibitory), or could simply reflect an after-effect of the excitatory response produced, for example, by a $\mathrm{K}(\mathrm{Ca})$ channel activated by calcium entry during the excitatory response. Overall, the data are consistent with the hypothesis that complex-spiking units, presumed to be cartwheel cells, convey inhibitory multimodal information to DCN principal cells.

unit); no features of the PF response vary consistently with BF. $B$, Latencies to the excitatory component of complex-spiking units ( filled bars) and the long-latency inhibitory component of type IV units (open bars). $C$, Relative amplitudes of the response components (see legend) as a function of PF stimulus pulse number. This figure shows the average ratio of the amplitudes of these features at pulses 1,3 , and 4 relative to their amplitudes in response to pulse 2. Error bars represent SEM; the numbers of units included are as follows: 15 complex-spiking units and 16 type IV units. All averages at pulses 1, 3, and 4 are significantly different from $1(p<0.01)$. 


\section{Complex-spiking units are cartwheel cells}

The extracellularly recorded complex-spiking units in this study are likely to be cartwheel cells for several reasons. First, all the units were located in the superficial layers of the DCN (first column of Table 1), consistent with the anatomic location of cartwheel cells (Brawer et al., 1974; Wouterlood and Mugnaini, 1984; Berrebi and Mugnaini, 1991). Second, the bursts of spikes termed complex-spikes in this (Fig. $2 A$ ) and other studies (Waller and Godfrey, 1994; Parham and Kim, 1995) are likely to be the extracellular equivalents of intracellularly recorded complex spikes obtained from identified cartwheel cells in DCN (Zhang and Oertel, 1993; Manis et al., 1994; Ding et al., 1996). The in vitro intracellular recording and labeling studies in guinea pig and mouse DCN suggest that complex-spiking behavior is associated exclusively with cartwheel cells (Zhang and Oertel, 1993; Manis et al., 1994). Finally, the complex-spiking units are typically only weakly responsive to sound (Table 1, Fig. 2B,C) (Parham and Kim, 1995), consistent with the anatomic observation that cartwheel cells do not appear to receive direct type I primary afferent input (Brown and Ledwith, 1990).

\section{Excitatory responses in cartwheel cells}

Activation of the granule cells, whether orthodromic by stimulating the MSN or antidromic by stimulating the PFs, strongly excites presumed cartwheel cells (Figs. 3, 5). It seems clear that this excitatory response reflects direct granule cell excitation of the cartwheel cells, because the PF system is the only known excitatory pathway in the superficial DCN (Oliver et al., 1983; Osen et al., 1995). Furthermore, the latency of the excitatory peak (column 3 of Table 2) corresponds to the latency of the EP. It has been argued, based on a current-source density analysis of the EPs, that the EP is produced by postsynaptic currents in the apical dendrites of pyramidal and cartwheel cells; thus, its onset provides a convenient marker for the arrival of the PF volley at the recording site (Young et al., 1995).

One difference between the excitatory responses elicited by MSN and PF stimulation is that MSN stimulation tends to induce complex-spike discharges, whereas PF stimulation tends to induce only simple spikes. Their response with complex spikes to granule cell activation distinguishes DCN cartwheel cells from cerebellar Purkinje cells. Cerebellar Purkinje cells respond with only simple spikes to granule cell stimulation; they produce complex spikes in response to climbing fiber stimulation (Eccles et al., 1967). Complex spikes in both DCN cartwheel cells and cerebellar Purkinje cells are mediated by calcium currents (Llinas and Sugimori, 1980a,b; Molitor and Manis, 1996), so the difference in complexspiking frequency between MSN and PF stimulation probably occurs because MSN stimulation produces more calcium current than PF stimulation. The larger calcium currents could come about because MSN stimulation activates more granule cells; however, it could also occur if orthodromic activation of the granule cells from the MSN activates cell types that are not activated by antidromic PF stimulation. These unknown cell types could then serve a role analogous to the climbing fibers in cerebellum. Unfortunately, current evidence argues against one possible candidate, the unipolar brush cell, which does not seem to receive somatosensory mossy fiber input (Wright and Ryugo, 1996).

A second difference between MSN and PF stimulation is that in the former, a reversal in the amplitude of the excitatory component is observed at the second pulse. That is, the amplitude of the excitatory response with MSN stimulation drops significantly between the first and second pulses and increases for subsequent pulses, whereas the size of the excitatory response monotonically increases with PF stimulation. The monotonic increase over pulses two to four in both responses likely reflects facilitation at the PF to cartwheel cell synapse, similar to that observed between granule cells and DCN principal cells (Manis, 1989) and that at granule cell synapses in cerebellum (Eccles et al., 1967). The large decrease from pulse one to two with MSN stimulation suggests an additional mechanism that must occur at or before the granule cells because it is not seen with PF stimulation. A reduction in the responsiveness of granule cells between the first and second pulse, perhaps attributable to refractoriness or feedback inhibition from Golgi cells, as in the cerebellum (Eccles et al., 1967), could account for this effect. Alternatively, the large firstpulse response could be a response to the additional cell type hypothesized in the previous paragraph, if that cell type showed strong adaptation after the first pulse.

\section{Functional implications of the granule cell circuitry}

The granule cell-associated circuitry of the superficial DCN integrates information across several sensory modalities and predominantly produces inhibition in the principal cells. Results from this study suggest that this inhibition is mediated, in large part, by cartwheel cells. The purpose of this system is not yet clear. One possibility is that the DCN may be involved in early sound source localization. In the cat, the filtering properties of the pinna produce narrow spectral notches that can provide sufficient cues to localize sounds because the notch frequencies depend on the location of the sound source relative to the pinna (Musicant et al., 1990; Rice et al., 1992). DCN principal cells are sensitive to such notches (Young et al., 1992; Nelken and Young, 1994). Clearly, input from the somatosensory and potentially the vestibular systems about the orientation and movement of the pinna and head needs to be integrated with the detection of these notches to allow accurate estimation of the location of the sound source. Perhaps the granule cell system, through the cartwheel cells, serves a role in this process.

\section{REFERENCES}

Berrebi AS, Mugnaini E (1991) Distribution and targets of the cartwheel cell axon in the dorsal cochlear nucleus of the guinea pig. Anat Embryol 183:427-454.

Berrebi AS, Morgan JI, Mugnaini E (1990) The Purkinje cell class may extend beyond the cerebellum. J Neurocytol 19:643-654.

Brawer JR, Morest DK, Kane EC (1974) The neuronal architecture of the cochlear nucleus of the cat. J Comp Neurol 155:251-300.

Brown MC, Ledwith JV (1990) Projections of thin (type-II) and thick (type-I) auditory-nerve fibers into the cochlear nucleus of the mouse. Hear Res 49:105-118.

Burian M, Gestoettner W (1988) Projection of primary vestibular afferent fibres to the cochlear nucleus in the guinea pig. Neurosci Lett 84:13-17.

Davis KA, Miller RL, Young ED (1996) Effects of somatosensory and parallel-fiber stimulation on neurons in dorsal cochlear nucleus. J Neurophysiol 76:3012-3024.

Ding J, Benson TE, Voigt HF (1996) Intracellular recordings of cartwheel and giant cells in dorsal cochlear nucleus (DCN) of unanesthetized, decerebrate gerbils (Meriones unguiculatus). Soc Neurosci Abstr 20:136.

Eccles JC, Ito M, Szentágothai J (1967) The cerebellum as a neuronal machine. New York: Springer.

Golding NL, Oertel D (1995) Evidence that cartwheel cells of the dorsal cochlear nucleus excite other cartwheel cells and inhibit principal cells with the same neurotransmitter, glycine. Soc Neurosci Abstr 21:399.

Golding NL, Oertel D (1996) Context-dependent synaptic action of glycinergic and GABAergic inputs in the dorsal cochlear nucleus. J Neurosci 16:2208-2219.

Itoh K, Kamiya H, Mitani A, Yasui Y, Takada M, Mizuno N (1987) 
Direct projections from the dorsal column nuclei and the spinal trigeminal nuclei to the cochlear nuclei in the cat. Brain Res 400:145-150.

Llinas R, Sugimori M (1980a) Electrophysiological properties of in vitro purkinje cell somata in mammalian cerebellar slices. J Physiol (Lond) 305:171-195.

Llinas R, Sugimori M (1980b) Electrophysiological properties of in vitro purkinje cell dendrites in mammalian cerebellar slices. J Physiol (Lond) 305:197-213.

Lorente de Nó R (1981) The primary acoustic nuclei. New York: Raven.

Manis PB (1989) Responses to parallel fiber stimulation in the guinea pig dorsal cochlear nucleus in vitro. J Neurophysiol 61:149-161.

Manis PB, Molitor SC (1996) $N$-Methyl-D-aspartate receptors at parallel fiber synapses in the dorsal cochlear nucleus. J Neurophysiol 76:1639-1656.

Manis PB, Spirou GA, Wright DD, Paydar S, Ryugo DK (1994) Physiology and morphology of complex spiking neurons in the guinea pig dorsal cochlear nucleus. J Comp Neurol 348:261-276.

Molitor SC, Manis PB (1996) Calcium signaling in dendrites of dorsal cochlear nucleus neurons. Soc Neurosci Abstr 22:647.

Mugnaini E, Morgan JI (1987) The neuropeptide cerebellin is a marker for two similar neuronal circuits in rat brain. Proc Natl Acad Sci USA 84:8692-8696.

Mugnaini E, Warr WB, Osen KK (1980) Distribution and light microscopic features of granule cells in the cochlear nuclei of cat, rat, and mouse. J Comp Neurol 191:581-606.

Musicant AD, Chan JCK, Hind JE (1990) Direction-dependent spectral properties of cat external ear: new data and cross-species comparisons. J Acoust Soc Am 87:757-781.

Nelken I, Young ED (1994) Two separate inhibitory mechanisms shape the responses of dorsal cochlear nucleus type IV units to narrowband and wideband stimuli. J Neurophysiol 71:2446-2462.

Oliver DL, Potashner SJ, Jones DR, Morest DK (1983) Selective labeling of spiral ganglion and granule cells with $\mathrm{D}$-aspartate in the auditory system of cat and guinea pig. J Neurosci 3:455-472.

Osen KK, Ottersen OP, Storm-Mathisen J (1990) Colocalization of glycine-like and GABA-like immunoreactivities: a semiquantitative study of individual neurons in the dorsal cochlear nucleus of cat. In: Glycine neurotransmission (Ottersen OP, Storm-Mathisen J, eds), pp 417-451. New York: Wiley.

Osen KK, Storm-Mathisen J, Ottersen OP, Dihle B (1995) Glutamate is concentrated in and released from parallel fiber terminals in the dorsal cochlear nucleus: a quantitative immunocytochemical analysis in guinea pig. J Comp Neurol 357:482-500.

Parham K, Kim DO (1995) Spontaneous and sound-evoked discharge characteristics of complex-spiking neurons in the dorsal cochlear nucleus of the unanesthetized decerebrate cat. J Neurophysiol 73:550-561.

Rice JJ, May BJ, Spirou GA, Young ED (1992) Pinna-based spectral cues for sound localization in the cat. Hear Res 58:132-152.

Sokolich, WG (1977) Improved acoustic system for auditory research. J Acoust Soc Am 62:12a.

Waller HJ, Godfrey DA (1994) Functional characteristics of spontaneously active neurons in rat dorsal cochlear nucleus in vitro. J Neurophysiol 71:467-478.

Weedman DL, Pongstaporn T, Ryugo DK (1996) Ultrastructural study of the granule cell domain of the cochlear nucleus in rats: mossy fiber endings and their targets. J Comp Neurol 369:345-360.

Wouterlood FG, Mugnaini E (1984) Cartwheel neurons of the dorsal cochlear nucleus. A Golgi-electron microscopic study in the rat. J Comp Neurol 227:136-157.

Wright DD, Ryugo DK (1996) Mossy fiber projections form the cuneate nucleus to the dorsal cochlear nucleus of rat. J Comp Neurol 365:159-172.

Young ED (1980) Identification of response properties of ascending axons from dorsal cochlear nucleus. Brain Res 200:23-38.

Young ED (1984) Response characteristics of neurons of the cochlear nuclei. In: Hearing science, recent advances (Berlin CI, ed), pp 423 460. San Diego: College Hill.

Young ED, Spirou GA, Rice JJ, Voigt HF (1992) Neural organization and responses to complex stimuli in the dorsal cochlear nucleus. Philos Trans R Soc Lond [Biol] 336:407-413.

Young ED, Nelken I, Conley RA (1995) Somatosensory effects on neurons in dorsal cochlear nucleus. J Neurophysiol 73:743-765.

Zhang S, Oertel D (1993) Cartwheel and superficial stellate cells of the dorsal cochlear nucleus of mice: intracellular recordings in slices. J Neurophysiol 69:1384-1397.

Zhang S, Oertel D (1994) Neuronal circuits associated with the output of the dorsal cochlear nucleus through fusiform cells. J Neurophysiol 71:914-930. 